\title{
Nosocomial rotavirus gastroenterocolitis in a large tertiary paediatric hospital in Warsaw, 2006-2010
}

Aneta Nitsch-Osuch ${ }^{1}$, Ernest Kuchar², Anna Kosmala ${ }^{3}$, Katarzyna Życinska ${ }^{1}$, Kazimierz Wardyn ${ }^{1}$

'Department of Family Medicine, Medical University of Warsaw, Poland ${ }^{2}$ Department of Paediatric Infectious Diseases, Medical University of Wroclaw, Poland ${ }^{3}$ District Paediatric Hospital, Warsaw, Poland

Submitted: 7 March 2011

Accepted: 11 September 2011

Arch Med Sci 2013; 9, 3: 493-498

DOI: 10.5114/aoms.2013.33177

Copyright (c) 2013 Termedia \& Banach
Corresponding author: Aneta Nitsch-Osuch PhD Department of Family Medicine Warsaw Medical University 1a Banacha St, Block F 02-097 Warsaw, Poland Phone: +48225992190 E-mail: anitsch@amwaw.edu.pl

\begin{abstract}
Introduction: Rotaviruses are the leading cause of community-acquired and nosocomial gastroenterocolitis in children. There are limited data concerning the epidemiology of nosocomial rotavirus gastroenterocolitis (NRVG) in Central European countries, including Poland. The aim of our study was to analyse the epidemiology of NRVG in a large tertiary hospital in Warsaw.

Material and methods: We analysed retrospectively data of 63173 patients aged 0-18 years hospitalized in the period 2006-2010. Nosocomial rotavirus gastroenterocolitis was defined as acute gastroenterocolitis ( $>3$ loose, or looser than normal, stools in $24 \mathrm{~h}$ and/or vomiting), confirmed with rapid immunochromatographic test (BioMaxima, Poland), if symptoms developed $>48 \mathrm{~h}$ after admission.

Results: In total 575 cases of NRVG were diagnosed. The cumulative attack rate of NRVG was calculated as $0.91 \%$ (95\% Cl: 0.85-0.98\%). The incidence density was 2.05/1000 bed-days ( $95 \% \mathrm{Cl}: 0.19-0.22 / 1000$ bed-days). The mean proportion of NRVG among all rotavirus infections was $24 \%$. The highest rates of NRVG were noted at wards where the mean duration of hospital stay was longer than 5 days (General Paediatrics and Neonatal Pathology). Seventy-one percent of children with NRVG were younger than 2 years. The mean duration of hospital stay of children with NRVG was longer than the average duration of hospitalization (11.6 days vs. 4.6 days, $p<0.01$ ).

Conclusions: Our study showed a relevant incidence of NRVG, which can prolong the children's hospital stay. Limiting the number of NRVG is important to improve patients' safety and to avoid additional costs. Routine vaccination against rotavirus diseases could reduce the number of NRVG.
\end{abstract}

Key words: hospital infection, viral diarrhoea, epidemiology, children.

\section{Introduction}

Acute gastroenterocolitis is a common disease among children in both developed and developing countries [1]. Rotaviruses are the leading cause of gastroenterocolitis in children under 5 years worldwide and the leading cause of nosocomial diarrhoea [2]. It has been estimated that 4.5 million episodes of rotavirus gastroenterocolitis occur each year in the European Union (EU) [3] resulting in 72 000-77 000 hospitalizations in the 23 million under-fives living in this part of the world [4]. The median proportion of cases of nosocomial rotavirus gastroenterocolitis (NRVG) among all cases of hospitalization for rotavirus disease is estimated to be $21 \%$ [4]. 
Data available for seven European countries indicate that $2.5-11.8 \%$ of children aged less than 5 years may develop nosocomial rotavirus gastroenterocolitis, and the incidence density (RV cases per 1000 bed-days) ranges from 0.7 to $10 / 1000$ bed-days (Pediatric Rotavirus European Committee, PROTECT4).

Rotavirus is a non-enveloped double-stranded RNA virus, which is mainly transmitted by the faecal-oral route or direct contact, but it can occasionally be transmitted by droplets. The incubation period for rotavirus gastroenterocolitis is 1-3 days, which is followed by the sudden onset of watery diarrhoea, with high risk of dehydration, vomiting, abdominal pain and fever. Symptoms usually last 4-7 days [5]. The virus is stable in the environment. Transmission can occur through the ingestion of contaminated water (or other fluids) and food, and through contact with contaminated surfaces and objects. The hospital environment and hands of the health care staff have the potential to become contaminated and serve as a reservoir for the virus $[5,6]$.

Shared rooms, close physical contact between children in communal rooms or play areas (sharing of toys) between children and visitors such as parents and siblings, and uncontrolled fluids and bodily secretions also provide opportunities for infection spread $[5,6]$.

Asymptomatic rotavirus infection (present in $20-40 \%$ of cases) is a particular cause of concern. Patients and health care workers with asymptomatic infection may also spread the disease and this may be another reason for difficulties in prevention of rotavirus nosocomial infections and the underestimation of their incidence $[6,7]$.

At present, the number of studies on the incidence of nosocomial RV infections is limited and available data have some limitations. For NRVG different case definitions have been used in published studies, with occurrence of infection ranging from $48 \mathrm{~h}$ to $72 \mathrm{~h}$. The studies were often performed during the rotavirus season only (not the whole year), and the age groups were different (most studies described the NRVG incidence among children younger than 2-5 years) $[3,4,7]$.

Limited reports about nosocomial diarrhoea due to rotavirus, both from Poland and Central Europe, are available. Most papers describe the epidemiology of community-acquired rotavirus infections.

The aim of our study was to describe the burden and epidemiology of nosocomial rotavirus gastroenterocolitis among paediatric patients aged 0-18 years treated in one large tertiary paediatric hospital in Warsaw (Poland) in the 5-year period 2006-2010.

\section{Material and methods}

The retrospective study was conducted at the Paediatric Hospital dedicated to the Memory of Professor Bogdanowicz in Warsaw, Poland. The hospi- tal is the largest tertiary hospital and the oldest working one in Warsaw (it was built in 1959). The hospital provides primary, secondary and tertiary care facilities for about 11 500-12 500 children yearly and it has about 300 beds. General paediatrics, general and traumatic paediatric surgery and a range of specialist services including critical care, neurosurgery, neurology, allergology and pulmonology, ophthalmology and ENT are provided.

Both official statistical data obtained from hospital records (including number of hospitalized patients, number of person-days, average duration of a hospitalization and data collected by the Hospital Infection Team) and individual medical files of children aged 0-18 years with nosocomial gastroenterocolitis due to rotavirus infection were analysed. The authors obtained written ethical approval from the hospital management and hospital ethics committee before conducting the study.

The analysed period was from $1^{\text {st }}$ January 2006 to $31^{\text {st }}$ December 2010.

Acute gastroenterocolitis was defined as diarrhoea (> 3 loose, or looser than normal, stools in a 24-hour period) and/or vomiting. Gastroenterocolitis was considered hospital-acquired if symptoms developed $>48 \mathrm{~h}$ after admission. Community-acquired gastroenterocolitis was diagnosed if symptoms of diarrhoea were present at admission to the hospital. Rotaviral infection was diagnosed with a rapid immunochromatographic test (BioMaxima, Poland) characterized by $98 \%$ sensitivity and $96 \%$ specificity. Stool samples were taken from all symptomatic patients. The same methodology was used to describe an epidemiological situation of a hospital in previous years [8].

In order to calculate the attack rate (the incidence of NRVG among hospitalized children), the numerator was represented by the reported NRVG cases (as previously defined) and the denominator was represented by the total number of patients minus the ones with community-acquired gastroenterocolitis. The incidence density was also calculated: the numerator was represented by the number of detected NRVG cases and the denominator was represented by the number of total bed-days spent in the hospital. The 95\% confidence interval was calculated on the incidence.

We also studied the difference between the mean duration of hospitalization and mean hospital stay of children with NRVG.

\section{Statistical analysis}

The statistical analysis was carried out by comparing the mean length of hospital stay with a nonparametric Mann-Whitney test.

\section{Results}

In the 5-year period (2006-2010), a total of 63173 patients were hospitalized and there were 1790 cases 


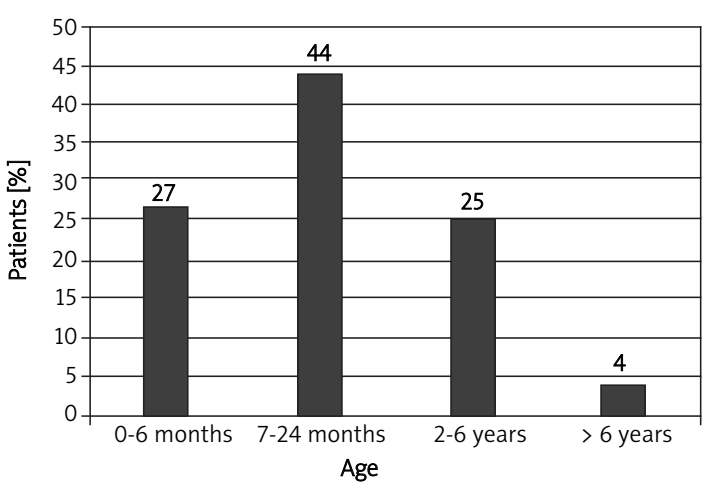

Figure 1. Age distribution of CARVG and NRVG

of community-acquired rotavirus gastroenterocolitis (CARVG) and 575 cases of nosocomial rotavirus gastroenterocolitis (NRVG). The median proportion of hospital-acquired rotavirus disease among all cases of hospitalization for rotavirus gastroenterocolitis was $24 \%$.

Most of the patients with NRVG were boys (304, $53 \%)$. Age of a child with NRVG ranged from 4 weeks to 12 years, and mean age of a patient with NRVG was 16.4 months (SD = 11.4 months). Nosocomial rotavirus infection was mostly diagnosed among patients aged 6 months -2 years (44\%); less common were infections among infants younger than 6 months (27\%) and children aged 2-6 years (25\%) - Figure 1.

The most common symptoms of nosocomial rotavirus infection were diarrhoea (present in 66\%), vomiting (in 68\%) and fever (in 64\%), while $80 \%$ of children required intravenous rehydration.

The mean duration of hospitalization of a child with NRVG was 11.6 days (SD $=0.4$ days) and the mean duration of non-complicated hospitalization 4.6 days ( $S D=0.3$ days). The difference between the mean duration of non-complicated hospital stay and the mean duration of hospital stay complicated by NRVG was statistically significant $(p<0.01)$.

Primary causes of hospitalization of the children with nosocomial rotavirus gastroenterocolitis were: respiratory tract infections (including pneumonia, bronchitis and otitis media) in $62 \%$ of patients, and urinary tract infection in $34 \%$ of patients. There were also other disorders including: myocarditis ( 2 cases), meningitis ( 5 cases), sepsis ( 9 cases), multiorgan trauma (5 cases), and observational and diagnostic procedures (8 cases).

The incidence density of NRVG ranged from 1.57/ 1000 person-days (in 2006) to $2.53 / 1000$ persondays (in 2009). The cumulative incidence density was $2.05 / 1000$ person-days ( $95 \% \mathrm{Cl}$ : 0.19-0.22/1000 person-days) (Table I).

The attack rate of NRVG (the proportion of hospitalized children developing NRVG) ranged from $0.78 \%$ (2010) to $1.09 \%$ (2007). The cumulative attack rate of NRVG was 0.91\% (95\% Cl: 0.85-0.98) (Table I).

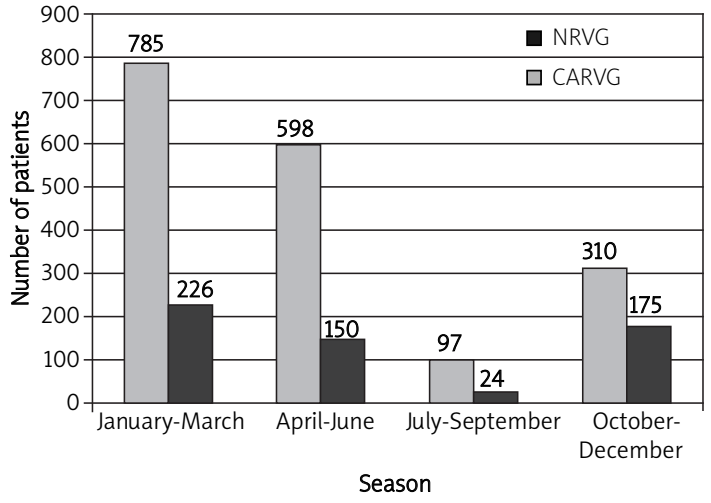

Figure 2. Seasonal distribution of CARVG and NRVG

Every year the majority of both CARVG and NRVG was diagnosed in autumn and winter months - from October to March, 1095 (62\%) and 401 cases (69\%), respectively - but the number of cases was also high in the third and second trimester of the year (Figure 2).

Most of the children with secondary rotavirus infection were hospitalized in the General Paediatric Ward - 249 patients (43\%), Neonatal Pathology - 137 (24\%) and Pulmonology and Allergology Ward - 143 (25\%), while a minority of the patients with NRVG (7\%) were located in ENT (15 cases), Neurosurgery (15 cases), Traumatic Surgery (7 cases), Ophthalmology (4 cases) and General Surgery (5 cases).

The highest cumulative attack rate and the highest cumulative incidence density were calculated for the General Paediatric Ward (respectively 5.6\% and 4.6/ 1000 person-days), Neonatal Pathology (respectively $1.6 \%$ and $2.7 / 1000$ person-days) and the Allergology and Pulmonology Ward (respectively $2.1 \%$ and 3.1/1000 person-days). The highest incidence rates were noted on wards where the average hospital stay is longer than 5 days (respectively: 9.9 days for General Paediatrics, 5.7 days for Neonatal Pathology and 6.1 days for Allergology and Pulmonology).

\section{Discussion}

Although studies on viral gastroenterocolitis in children have mainly focused on community-acquired infection, the importance of nosocomial rotavirus

Table I. Incidence density and attack rate of NRVG in 2005-2010

\begin{tabular}{|lcc|}
\hline Year & $\begin{array}{c}\text { Incidence density } \\
\text { per } \mathbf{1 0 0 0} \text { person-days }\end{array}$ & $\begin{array}{c}\text { Attack } \\
\text { rate [\%] }\end{array}$ \\
\hline 2006 & 1.57 & 0.81 \\
\hline 2007 & 2.23 & 1.09 \\
\hline 2008 & 2.0 & 0.9 \\
\hline 2009 & 2.53 & 1.08 \\
\hline 2010 & 1.95 & 0.78 \\
\hline Cumulative rate 2006-2010 & 2.05 & 0.91 \\
\hline
\end{tabular}


gastroenterocolitis and the potential for its prevention has been highlighted in several publications $[4,7-9]$. However, rotavirus infection contracted by hospitalized children should be a source of worry: the infection causes the child to stay longer in hospital, and there are additional economic and social costs (connected for example with absence from work of family members who assist the young patient - indirect costs) [10].

Our data show that the cumulative attack rate of nosocomial rotavirus gastroenterocolitis was $0.91 \%$. Polish data concerning the burden of nosocomial rotavirus infection are limited. It was estimated in a prospective study that $2 \%$ of hospitalized children younger than 7 years developed rotavirus diarrhoea [11]. It is noteworthy that in older Polish studies this rate was higher: $4.5 \%$ to $9 \%[12,13]$.

There are few papers from Central European countries describing the epidemiological situation in the aspect of nosocomial rotavirus infection, and available results are similar to ours. In a study conducted in the Slovak Republic, the attack rate of rotaviral nosocomial gastroenterocolitis was $0.65 \%$ (among children younger than 18 years) [14].

The attack rate of NRVG in our hospital is within lower limits typical for European countries. A 2006 review on NRVG incidence across Europe reported that NRVG accounts for $0.3-27.7 \%$ of all hospital admissions [7]. Results similar to ours were also described by Waisbourd-Zinman et al. from Israel: NRVG occurred in 1\% of all admissions [15].

The incidence density of NRVG in our study was $2.05 / 1000$ person-days and it was comparable with data from other papers in which the observation period covered the whole year: 1.6-2.6/1000 beddays (Austria) [16], 1.6/1000 bed-days (Germany) [17], 0.7/1000 bed-days (Switzerland) [17], 1.72/1000 bed-days (Slovak Republic) [14]. A 2006 review of NRVG incidence density across Europe reported that NRVG accounts for 1.6-15.8 per 1000 days of hospital stay [7].

The median proportion of hospital-acquired rotavirus infections among all hospitalized children with rotaviral gastroenteritis in our study was $24 \%$ and it was only slightly higher than the rate typical for European countries (21\%) [3]. Previous results from Poland, reported 20 years ago, indicated this rate to be higher: $39 \%$ [13].

The highest attack rate and the incidence density were reported in the wards where the mean duration of hospitalization was longer than 5 days: General Paediatrics, Neonatal Pathology and Allergology and Pulmonology. This corresponds well with results of previous studies which have proven that the risk of contracting NRVG increased significantly if the child stayed in hospital more than 5 days $(R R=2.8)$ [18], so the risk of NRVG increases with the length of hospital stay [14]. What is interesting, no NRVG was diagnosed among patients from the
Intensive Care Unit. This observation is in agreement with others: Ozer et al. found nosocomial infection in $20.1 \%$ of patients, but these were only respiratory tract infection, urinary tract infections, bloodstream, wound and central nervous system infections [19]. Similar results were described by Kallel et al.: nosocomial infections in $16.9 \%$ of patients, mainly ventilator-associated pneumonia and bloodstream infection [20].

In our study $69 \%$ of NRVG occurred in the autumn and winter season, which is comparable with other data available in the literature [7, 14, 21]. This is not a surprising result because rotavirus disease incidence is highest in this season of the year in the general population. That is one more proof that children with community-acquired diarrhoea or vomiting should be isolated and taken care of by dedicated personnel in order to limit the spread of the disease. Since the transmission of rotavirus infection occurs mainly by contact, it is very important to insist on correct hand washing both by medical staff and relatives of the hospitalized child.

Hospital acquired rotavirus diseases was reported in our study mainly among children aged from 6 months to 2 years while in the literature we can find the opinion that nosocomial disease is more typical for infants younger than 6 months [7]. However, some authors have observed that the mean age of children with NRVG is 9 months or the children with community-acquired rotavirus disease were 9 months older than children with nosocomial infection [14]. The reduced incidence of NRVG among children younger than 6 months may be explained both by breastfeeding and by the presence of transplacental transferred maternal anti-RV antibodies in younger infants [13]. Generally, the peak incidence of nosocomial rotavirus gastroenterocolitis is reported to be less than 2 years of life $[13,18]$.

Symptomatology of NRVG in our patients was typical, with predominating symptoms of diarrhoea and vomiting, but it is noteworthy that $80 \%$ of patients required intravenous rehydration, which may be an indirect proof of the severity of the disease and may lead to another hospital-acquired infection due to prolonged use of intravenous access.

The mean length of hospital stay of children with NRVG (11.6 days) was significantly longer than the average duration of treatment in the hospital (4.6 days). This observation is consistent with the results of other studies which described longer hospital stay of children with NRVG (7.3 days [14], 8.1 days [21], 9 days [15]). The prolonged hospital stay of children with nosocomial rotavirus infection may generate higher costs and influence the quality of life of a hospitalized child. It may also predispose to a colonisation or infections caused by different drug resistant bacteria $[22,23]$.

Primary causes of admission to the hospital among children with secondary nosocomial rotavirus infec- 
tion were respiratory tract infections and urinary tract infections. These results are also consistent with literature data [14]. It must be clear for the primary care physicians that children should be referred to the hospital only in severe cases which may not be treated at home or in out-patient settings. Nosocomial rotavirus infections coincide with the winter peak of childhood virus infections (e.g. respiratory syncytial virus and influenza virus infections), which explains the high incidence of NRVG among patients from the General Paediatrics Ward, Neonatal Pathology Ward and Pulmonary and Allergology Ward.

Our study was a retrospective descriptive study with all its limitations, hence more prospective studies in this field are required. Our data may be underestimated because of two reasons: a possibility of asymptomatic nosocomial rotavirus infection and lack of follow-up several days after hospital discharge. Some patients (up to 30\%) may develop symptoms of NRVG after discharge and these cases might be missing in hospital records $[6,9,14]$.

The results of our study are also limited by the lack of available data concerning breast feeding. Breastfeeding could reduce gastrointestinal infections as breast milk contains lactadherin, secretory IgA, lymphocytes B and T, bacterial lactoferrin, oligosaccharides and glycans that protect intestinal epithelium against pathogens. It should be underlined that in the hospital where the analysis was conducted, mothers were strongly recommended to continue breastfeeding and were able to stay with the child in the hospital in order to take care of them. However, the specific role of breastfeeding in the prevention of rotavirus diarrhoea has not been well established, although it is generally considered to at least reduce the severity of the disease. Some studies have found a lower incidence of rotavirus diarrhoea in infants who received breast milk [24, 25], while others did not prove the protective effect [26-28].

Another limitation of our study may be the lack of information concerning how many children were vaccinated against rotavirus gastroenterocolitis. In 2007 the WHO recommended the inclusion of rotavirus vaccination in the national immunization schedules. Evidence of the effectiveness of immunization programmes is available for the two existing RV vaccines, both in industrialized and in developing countries [29-31]. Over the last few years, several studies have been performed to assess the cost-effectiveness of routine infant rotavirus vaccination and reported inconsistent and varying results [32]. For example, Goossens et al. [33] and Rozenbaum et al. [34] concluded that mass vaccination against rotavirus gastroenterocolitis can be attractive from both an economic and a health care point of view, while a more recent paper by Mangen et al. [35] stated that vaccination cannot be considered cost-effective. According to Diez-Domingo et al., uni- versal vaccination of Spanish children would avoid more than 2000 cases of nosocomial rotavirus gastroenterocolitis, saving 22 million euro from the national health system perspective and 38 million euro from the societal perspective [36].

Reported RV vaccine coverage in European countries varies from $35 \%$ to $85 \%$ [37], while in the USA it is as high as $70 \%$ in some states [38]. In Poland this vaccination is not mandatory, so it is not paid by the government but it may be bought by patients (or parents) with private funds. The RV vaccine coverage in our country is unknown because official data report only the number of given doses (not the number of vaccinated children). Data from single studies indicated that the RV vaccine coverage is low $(<30 \%)$ [39]. The universal use of rotavirus vaccine could definitely have potential to protect against and to control NRVG among children.

In conclusion, our study showed that the relevant incidence of nosocomial rotavirus gastroenterocolitis in Poland is similar to other European countries and increases with the length of the children's stay in hospital. Limiting the number of nosocomial rotavirus infections may be an important factor to improve patients' safety as well as to avoid additional health costs. Additional data on the incidence of NRVG are useful to evaluate the risks linked with healthcare, to plan the use of available resources, and to estimate healthcare costs. Universal rotavirus vaccination could reduce the incidence of nosocomial rotavirus gastroenterocolitis.

\section{References}

1. Parashar UD, Burton A, Lanata C, et al. Global mortality associated with rotavirus disease among children in 2004. $J$ Infect Dis 2009; 200 (Suppl): S9-15.

2. Parashar UD, Gibson CJ, Breese JS, et al. Rotavirus and severe childhood diarrhea. Emerg Infect Dis 2006; 12: 304-6.

3. Verstraeten T, van den Bosch W. Burden of rotavirus disease in the new European Union (poster). Presented at $23^{\text {rd }}$ ESPID Congress, Valencia, Spain 2005.

4. The paediaric burden of rotavirus diseases in Europe. Pediatric ROTavirus European CommitTee (PROTECT). Epidemiol Infect 2006; 134: 908-16.

5. Cone R, Mohan K, Thouless M, Corey L. Nosocomial transmission of rotavirus infection. Pediatr Infect Dis J 1988, 7: 103-9.

6. Posfay KM, Zerr DM, Pittet D. Infection control in pediatrics. Lancet Infect Dis 2008; 8: 19-31.

7. Gleizes O, Desselberger U, Tatochenko V, et al. Nosocomial rotavirus infection in European countries: a review of the epidemiology, severity and economic burden of hospital-acquired rotavirus disease. Pediatr Infect Dis 2006; 25 (Suppl 1): S12-21.

8. Nitsch-Osuch A, Kuchar E, Kosmala A, Życińska K, Wardyn K. Nosocomial gastroenterocolitis in children hospitalized primary due to respiratory infections. Adv Exp Med Biol 2013; 755: 267-74.

9. Festini F, Cochi P, Mambretti D, et al. Nosocomial rotavirus gastroenterocolitis in pediatric patients: a multi-center prospective cohort study. BMJ Infectious Diseases 2010; 10: 1-8. 
10. Jacobs P, Shane L, Fassbender K, et al. Economic analysis of rotavirus-associated diarrhea in the metropolitan Toronto and Peel regions of Ontario. Can J Infect Dis 2002; 13: 167-74.

11. Sulik A, Ołdak E, Rozkiewicz D, et al. Prospective study of rotavirus infections in children hospitalized at the Clinic of Pediatric Infectious Diseases in Białystok in 2003. Przegl Epidemiol 2004; 58: 475-81.

12. Dziechciarz P, Wernik T, Zalewski T. Nosocomial rotavirus infections. Pediatr Pol 1997; 72: 499-502.

13. Mrukowicz J, Krobicka B, Duplaga M. Epidemiology and impact of rotavirus diarrhea in Poland. Acta Paediatr 1999; 426: 53-60.

14. Stefcovicova M, Simurka P, Jurackova L, et al. Nosocomial rotavirus gastroenterocolitis in pediatric department. Centr Eur J Publ Health 2008; 16: 12-6.

15. Waisbourd-Zinman O, Ben-Ziony S, Solter E, et al. Hospitalizations for nosocomial gastroenterocolitis in a tertiary pediatric center: a 4-year prospective study. Am J Infect Control 2009; 37: 465-9.

16. Fruhwirth M. Economic impact of community and nosocomially acquired rotavirus gastroenteritis in Austria. Pediatr Infect Dis J 2001; 20: 184-8.

17. Fruhwirth $M$. International variation in disease burden of rotavirus gastroenteritis in children with community- and nosocomially acquired infection. Pediatr Infect Dis J 2001; 20: 784-91.

18. Muhsen K, Shulman L, Rubinstein U, et al. Incidence, characteristics, and economic burden of rotavirus gastroenterocolitis associated with hospitalization of Israeli children < 5 year of age, 2007-2008. J Infect Dis 2000; Suppl 1: S254-63.

19. Ozer B, Ozbakis A, Duran N, et al. Evaluation of nosocomial infections and risk factors in critically ill patients. Med Sci Monit 2011; 25: PH17-22.

20. Kallel H, Dammak H, Bahloul M, et al. Risk factors and outcomes of intensive care unit-acquired infections in a Tunisian ICU. Med Sci Monit 2010; 18: PH 69-75.

21. Gutierrez-Gimeno MV, Martin-Moreno JM, Diez-Domingo J, et al. Nosocomial rotavirus gastroenterocolitis in Spain: a multicenter prospective study. Pediatr Infect Dis J 2010; 29: 23-7.

22. Sadowska-Krawczenko I, Jankowska A, Kurylak A. Heathcare-associated infections in a neonatal intensive care unit. Arch Med Sci 2012; 8: 854-8.

23. Sękowska A, Gospodarek E, Kamińska D. Antimicrobial susceptibility and genetic similarity of ESBL-positive Klebsiella pneumoniae strains. Arch Med Sci 2012; 8: 993-7.

24. Naficy AB, Abu-Elyazeed R, Holmes JL, et al. Epidemiology of rotavirus diarrhea in Egyptian children and implications for disease control. Am J Epidemiol 1999; 150: 770-7.

25. Plenge-Bong A, Sato-Ramirez N, Karmaus W, et al. Breastfeeding protects against acute gastroenterocolitis due to rotavirus in infants. Eur J Pediatr 2002; 169: 1467-71.

26. Golding J, Emmett PM, Rogers IS. Gastroenterocoilitis, diarrhea and breast feeding. Early Hum Dev 1997; 49 Sup pl: S83-103.

27. Misra SM, Sabui TM, Basu SM, et al. A prospective study of rotavirus diarrhea in children younger than 1 year of age. Clin Pediatr (Phila) 2007; 46: 683-8

28. Wobudeya E, Bachou H, Karamagi C, et al. Breastfeeding and the risk of rotavirus diarrhea in hospitalized infants in Uganda: a matched case control study. BMJ Pediatrics 2011; 11: 17-22.

29. Vesikari T, Matson DO, Dennehy P, et al. Safety and efficacy of a pentavalent human-bovine (WC3) reassortant rotavirus vaccine. N Engl J Med 2006; 354: 23-33.
30. Ruiz-Palacios GM, Pérez-Schael I, Velázquez FR, et al. Safety and efficacy of an attenuated vaccine against severe rotavirus gastroenteritis. N Engl J Med 2006; 354: 11-22.

31. Madhi SA, Cunliffe NA, Steele D, et al. Effect of human rotavirus vaccine on severe diarrhea in African infants. $\mathrm{N}$ Engl J Med 2010; 362: 289-98.

32. Postma MJ. Public health economics of vaccines in the Netherlands: methodological issues andapplication. J Publ Health 2008; 16: 267-71.

33. Goossens LM, Standaert B, Hartwig N, et al. The cost-utility of rotavirus vaccination with Rotarix (RIX 4414) in the Netherlands. Vaccine 2008; 26: 1118-27.

34. Rozenbaum MH, Mangen MJ, Giaguinto C. Cost-effec tiveness of rotavirus vaccination in the Netherlands: the results of a consensus model. BMJ Public Health 2011; 11: 462-71.

35. Mangen MJ, Duynhoven YT, Vennema $H$, et al. Is it costeffective to introduce rotavirus vaccination in the Dutch national immunization program? Vaccine 2009; 27: 7222-7.

36. Diez-Domingo J, Surinach NL, Alcalde NM. Burden of paediatric rotavirus gastroenterocolitis (RVGE) and potentia benefits of a universal rotavirus vaccination programme with a pentavalent vaccine in Spain. MBC Public Health 2010: 10: 469-76.

37. Parashar U. Uptake and impact of Rotavirus vaccines in U.S. children. $14^{\text {th }}$ International Congress on Infectious Diseases; March 9-12, 2010; Miami.

38. Vesikari T, Sutherland D, Jackson AE. Report of the 'European Expert Meeting on Rotavirus Vaccination', Tampere, Finland, 19-20 May 2009.

39. Pokorna-Kałwak D, Gwiazda E, Mastalerz-Migas A, et al. Rotavirus vaccine coverage in one primary care setting. Family Med Prim Care Rev 2009; 11: 458-60. 